\title{
Immunotherapy for colon cancers: The beginning of a new story
}

\author{
Saber Boutayeb*, Ibrahim El Ghissassi, Hind Mrabti and Hassan Errihani \\ National Institute of Oncology, Mohammed V University, Rabat, Morocco
}

MSI (Microsatellite instability) is a molecular marker of defective DNA mismatch repair that is detected in approximately $15 \%$ of sporadic colon cancers in general and $5 \%$ of metastatic colon cancers $[1,2]$. The early stages colon cancers with MSI-H (High) have better stage-adjusted survival rates compared with MSS tumors. Moreover, the data analyses of randomized adjuvant therapy trials have suggested that MSI-H tumors do not benefit from treatment with 5-fluorouracil. A pooled analysis of these studies, confirmed the prognostic and predictive impact of MSI status in colon cancer [3].

Basing on these findings, the MSI status is used in clinical decisionmaking for adjuvant chemotherapy $[4,5]$.

The data for the stage IV colon cancers showed that patients who have MSI-H tumors (Also called dMMR) are less likely to respond to conventional chemotherapy. The anatopathologic analyses showed that the tumors MSI-H are highly immunogenic [6]. One of the most important characteristic of the immune system is its ability to tell between normal cells in the body and those it sees as "foreign" [7].

Once the "foreign" is detected, the immune system uses receptors on immune cells that need to be activated (or inactivated) to start an immune response. These molecules are called "checkpoints". PD-1 is one of the major checkpoint protein on the $\mathrm{T}$ cells [7]. The cancer cells use often these checkpoints to prevent their attack by the immune system. Cancer cells often develop ways to use these checkpoints to avoid being attacked by the immune system. Some cancer subtypes have high expression of PD-L1 (Ligand of PD-1), which helps them evade immune attack [7].

Monoclonal antibodies that target either PD-1(Nivolumab, Pembrolizuma) or PD-L1(Atezolizumab, Avelumab...) can block this interaction and boost the immune response against cancer cells. These drugs have demonstrated an important activity in the treatment of several types of cancer, including melanoma of the skin, non-small cell lung cancer, kidney cancer, bladder cancer, head and neck cancers, and Hodgkin lymphoma [7].

To evaluate how the colorectal metastatic cancer responds to nivolumab (Anti PD-1), a phase 2 trial (CheckMate 142) was conducted. The results of this study were recently published online July 19 in Lancet Oncology. Researchers in eight countries recruited 74 adults with histologically confirmed metastatic MSI-H colorectal cancers. Patients either were intolerant to at least one past line of chemotherapy or had progressive cancer despite treatment. Fifty-four percent $(n=40)$ were heavily pretreated and had received three or more past lines of chemotherapy [7].

Participants received $3 \mathrm{mg} / \mathrm{kg}$ intravenous nivolumab monotherapy every 2 weeks until tumor progression, death or unacceptable toxicity.
The primary endpoint was investigator-assessed objective response according to Response Evaluation Criteria in Solid Tumors (RECIST) guidelines. Response was evaluated both by local investigators and central reviewers [8].

During a median follow-up of 12 months, $31.1 \%$ of patients ( $\mathrm{n}=$ 23 of $74 ; 95 \%$ confidence interval [CI], 20.8\% to $42.9 \%$ ) reached the primary endpoint, all of whom showed partial response. Among the responders, eight (8) patients had responses that exceed 12 months.

The median progression-free survival was 14.3 months (95\% CI, 4.30 months to not estimable). Twelve-month overall survival was $73 \%$ (95\% CI, $62 \%$ to $82 \%$ ). Additional analyses showed that the response to nivolumab is not correlated to programmed cell death (PD) ligand 1 expression and biomarkers for colorectal cancer (RAS and RAF status) [8].

Concerning the serious adverse events, forty-one percent of patients $(n=31)$ experienced grade 3 adverse events, and 14\% ( $n=10)$ developed grade 4 adverse events. But no toxic death occurred. The most common toxicities were increased lipase (6 patients [8\%]) and amylase (2 patients [3\%]). Seven percent of patients discontinued nivolumab because of drug-related adverse events [8].

According these findings, Nivolumab has just been approved by the US Food and Drug Administration (FDA) for this indication. As we could expect, the decision algorithm for MSI-H metastatic colorectal cancer is rapidly evolving. The National Comprehensive Cancer Network (NCCN) panel recommends nivolumab as a category 2A treatment option for patients with MSI-H colorectal cancer as second- or third-line therapy. In the same way, The NCCN practice guidelines also recommend universal MSI testing for all patients [9].

\section{References}

1. Grady WM, Carethers JM (2008) Genomic and epigenetic instability in colorectal cancer pathogenesis. Gastroenterology 135: 1079-1099. [Crossref]

2. Gafa R, Maestri I, Matteuzzi M, Santini A, Ferretti S, et al. (2000) Sporadic colorectal adenocarcinomas with high-frequency microsatellite instability. Cancer 89: 2025 2037. [Crossref]

3. Sinicrope FA, Rego RL, Halling KC, Foster N, Sargent DJ, et al. (2006) Prognostic impact of microsatellite instability and DNA ploidy in human colon carcinoma patients. Gastroenterology 131: 729-737. [Crossref]

Correspondence to: Boutayeb Saber, National Institute of Oncology, Mohammed V University, Rabat, Morocco, Tel: +212 5372-72750; E-mail: Saber.boutayeb@ um5s.net.ma

Received: December 06, 2017; Accepted: December 22, 2017; Published: December 26, 2017 
4. Halling KC, French AJ, McDonnell SK, Burgart LJ, Schaid DJ, et al. (1999) Microsatellite instability and $8 \mathrm{p}$ allelic imbalance in stage B2 and C colorectal cancers. J Natl Canc Inst 91: 1295-1303. [Crossref]

5. Lanza G, Gafa R, Santini A, Maestri I, Guerzoni L, et al. (2006) Immunohistochemical test for MLH1 and MSH2 expression predicts clinical outcome in stage II and III colorectal cancer patients. J Clin Oncol 24: 2359-2367. [Crossref]

6. Nash GM, Gimbel M, Cohen AM, Zeng ZS, Ndubuisi MI, et al. (2010) KRAS mutation and microsatellite instability: two genetic markers of early tumor development that influence the prognosis of colorectal cancer. Ann Surg Oncol 17: 416-424. [Crossref]
7. Li B, Chan HL, Chen P (2017) Immune checkpoint inhibitors: basics and challenges. Curr Med Chem. [Crossref]

8. Overman MJ, McDermott R, Leach JL, Lonardi J, Lenz HJ, et al. (2017) Nivolumab in patients with metastatic DNA mismatch repair-deficient or microsatellite instabilityhigh colorectal cancer (CheckMate 142): an open-label, multicentre, phase 2 study. Lancet Oncol 8: 1182-1191. [Crossref]

9. https://www.nccn.org/professionals/physician_gls/PDF/colon.pdf

Copyright: $\odot 2017$ Boutayeb S. This is an open-access article distributed under the terms of the Creative Commons Attribution License, which permits unrestricted use, distribution, and reproduction in any medium, provided the original author and source are credited. 